\title{
Tungiasis. Presentación de un caso clínico
}

\author{
Tungiasis: a case report
}

\author{
Dr. Kim Sung Hoon ${ }^{a}$, Dra. María Florencia Fernández ${ }^{a}$ Dra. María Marta Bujána, \\ Dra. Andrea Bettina Cervinia, Dr. Jorge Laffargue y Dr. Adrián Martin Pierini ${ }^{a}$
}

\section{RESUMEN}

La tungiasis es una parasitosis cutánea originaria de América causada por Tunga penetrans. Se caracteriza por lesiones papulares, negruzcas, únicas o múltiples, que suelen afectar los pies, principalmente en las zonas subungueales y periungueales. El diagnóstico de tungiasis se realiza por las características clínicas de las lesiones en un paciente proveniente de zonas endémicas. El tratamiento de elección es la extracción quirúrgica de la pulga y la aplicación de antibióticos tópicos.

Presentamos un caso de tungiasis en una paciente de 10 años de edad con múltiples lesiones en ambos pies, que fue tratada satisfactoriamente con ivermectina y extracción quirúrgica.

Palabras clave: Tunga penetrans, tungiasis, niños.

\section{SUMMARY}

Tungiasis, is a cutaneous parasitosis, native of America caused by Tunga penetrans. Infestations usually presents with black papular lesions, either single or multiple, most of them localized on the feet, mainly in the subungual and periungual areas. Diagnosis of tungiasis is based on the characteristic aspect of the lesions in a patient coming from an endemic area.

Surgical removal of the flea and application of a topical antibiotic is the standard treatment.

We describe a case of a 10-years-old girl, with multiple lesions localized on feet, who was succesfully treated with ivermectin and surgical removal of lesions.

Key words: tungiasis, Tunga penetrans, children.

\section{INTRODUCCIÓN}

La tungiasis es una endozoonosis originaria de América Central y del Sur, que causa en el hombre una ectoparasitosis transitoria, cuyo agente etiológico es la pulga hembra de Tunga penetrans (familia Siphonaptera). Este parásito invade la epidermis y produce lesiones características. ${ }^{1}$

Presentamos un caso de tungiasis múltiple en una niña de 10 años de edad.

a. Servicio de Dermatología.

Hospital de Pediatría "Prof. Dr. Juan P. Garrahan".

Ciudad Autónoma de Buenos Aires.

Correspondencia:

Dr. Kim Sung Hoon:

cachiksh@hotmail.com

Conflicto de intereses: Ninguno que declarar.

Recibido: 6-1-11

Aceptado: 20-4-11

\section{CASO CLÍNICO}

Paciente de sexo femenino de 10 años edad, oriunda de la provincia de Misiones, que consultó al servicio de Dermatología por presentar múltiples placas de aspecto verrugoso con numerosas pápulas negruzcas sobre su superficie, localizadas en los sitios de apoyo de ambos pies (punta de dedos, metatarso y talones), de reciente aparición (Figuras 1 y 2).

FIgURA 1. Múltiples pápulas negruzcas en región lateral de metatarso derecho

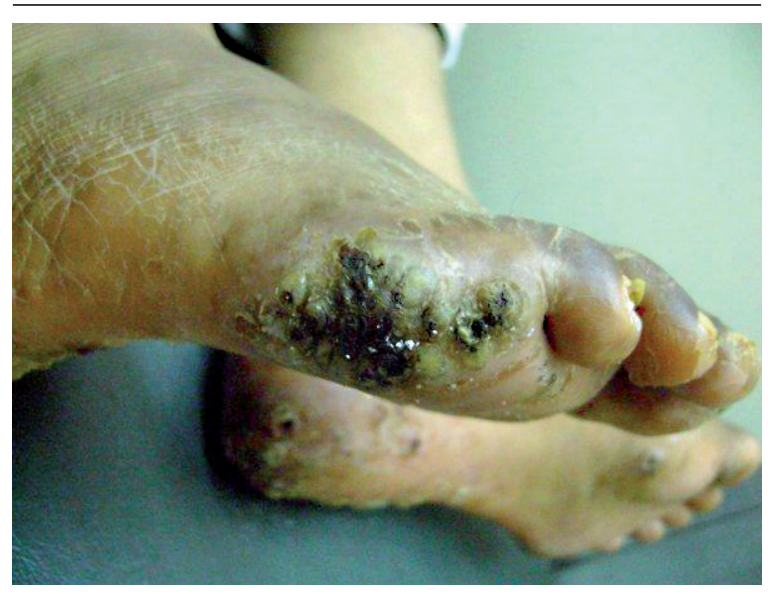

FIGURA 2. Pápulas blanquecinas con punto negro central en talón de pie derecho

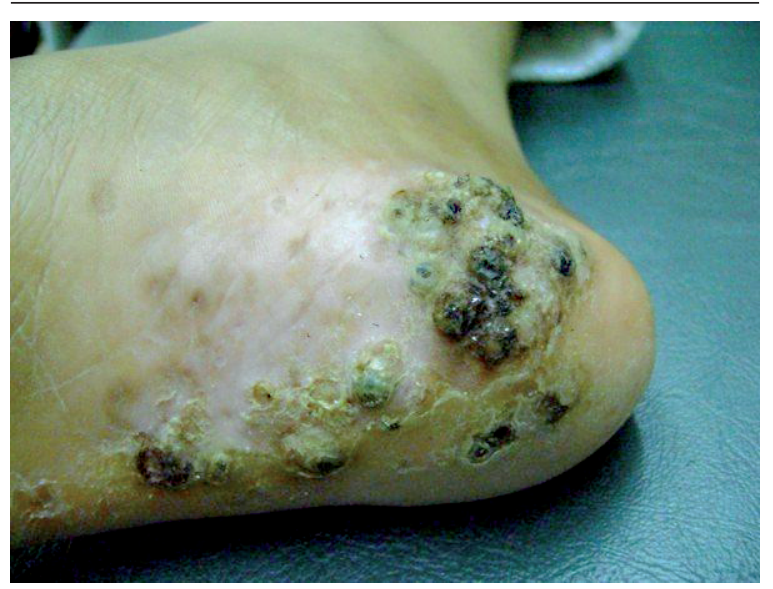


Las lesiones le ocasionaban dolor y dificultaban su deambulación. Realizada la extracción quirúrgica, se obtuvieron alrededor de 20 tungas y las muestras extraídas se enviaron al servicio de anatomopatología, que confirmó el diagnóstico de tungiasis. La paciente fue tratada con ivermectina vía oral $200 \mu \mathrm{g} / \mathrm{kg}$ como terapia coadyuvante, debido al gran compromiso cutáneo y la multiplicidad de lesiones. Las lesiones evolucionaron favorablemente con resolución completa.

\section{DISCUSIÓN}

La tungiasis es una infección cutánea causada por la pulga hembra de Tunga penetrans (familia Siphonaptera), que en el hombre ocasiona una ectoparasitosis transitoria. Es una endozoonosis originaria de América Central, América del Sur y el Âfrica subsahariana; se halla en áreas de suelo seco, arenoso y sombreado. ${ }^{1,2}$ En nuestro país se han registrado casos en Salta, Jujuy, Tucumán, Santiago del Estero, Santa Fe, Chaco, Formosa, Misiones y Corrientes. Esta pulga hematófaga no tiene especificidad por el huésped, por lo que puede afectar tanto al hombre como a diversos animales, como aves de corral, cerdos y perros, entre otros. ${ }^{1}$

La infección se ve favorecida por malos hábitos, como caminar descalzo, dormir en el suelo y tener una higiene deficiente; prevalece en niños de 5-14 años de edad, por sus hábitos de jugar en el suelo. ${ }^{3}$

La tunga se conoce también como nigua (Argentina, Venezuela), kuti, suthipique (Bolivia), ogrieye (Surinam), bicho de pe', bicho de porco (Brazil), chica (Colombia y Venezuela), sikka (Guyana), pique (Argentina, Chile, Uruguay y Paraguay), piqui (quechua), tii (tupí-guaraní), jigger, sand flea chigoe o burrowing flea (Estados Unidos)., 1,2

El ciclo vital del parásito es similar al de otros artrópodos y dura aproximadamente un mes, durante el cual la tunga pasa por cuatro estadios biológicos: huevo, larva, pupa y adulto. El ciclo comienza con la hembra fecundada, que penetra en la piel del huésped hasta la dermis papilar. Allí se alimenta de la sangre de éste y aumenta su tamaño hasta alcanzar 0,6-1,0 cm. Durante un lapso de 7-10 días expulsa diariamente entre 150 y 200 huevos a través de su orificio abdominal caudal y luego muere. Los huevos expulsados eclosionan a los 3-4 días y pasan al estado de larva, que luego de dos semanas se transforman en pupas y, posteriormente, en parásito adulto, con lo cual se completa el ciclo vital. ${ }^{1,3}$

La tunga suele parasitar los pies (talón, plan- tas, espacios interdigitales y zonas subungueales y periungueales) de quienes caminan descalzos en suelos arenosos o secos de tierra. Menos frecuentemente puede afectar otros sitios del cuerpo, como glúteos, tronco o manos. ${ }^{1,3}$

La tungiasis, al examen físico, se presenta con una tríada clínica: 1) punto negro central que corresponde a la cloaca; 2) alrededor de él, un halo translúcido que corresponde al abdomen lleno de huevos y 3) hiperqueratosis periférica que corresponde a una reacción por cuerpo extraño de la piel circundante. ${ }^{3}$

Las lesiones aumentan progresivamente de tamaño a medida que los huevos se acumulan en el saco gestacional, hasta alcanzar 5-10 mm de diámetro. El aparato masticatorio del parásito se encuentra ubicado hacia la dermis profunda, de cuyos vasos sanguíneos se alimenta. ${ }^{4}$

La infección, según el sitio de localización, puede ser asintomática, pruriginosa o dolorosa y ocasionar molestias al deambular. En otras ocasiones, los pacientes sólo refieren sensación de cuerpo extraño. ${ }^{1,4}$

Las complicaciones son: sobreinfección bacteriana de las lesiones, linfangitis e infartos ganglionares, o tétanos en pacientes no vacunados. En casos graves también pueden observarse autoamputaciones de los dedos. ${ }^{2,3}$

El diagnóstico de tungiasis es clínico, al visualizarse las típicas lesiones en un paciente con antecedentes de haber estado en un área endémica. Además, con una lupa, se puede observar una estructura marrón-amarillenta correspondiente al parásito, así como también sus múltiples huevos. En caso de dudas diagnósticas, puede realizarse un corte sobre la lesión y enviar el contenido extraído en solución fisiológica al laboratorio de parasitología, donde se identificarán al microscopio porciones de la tunga junto con numerosos huevos. ${ }^{1}$

Se deben considerar como diagnósticos diferenciales los nevos acrales eruptivos, el melanoma, las verrugas vulgares y la cromomicosis.

La extracción quirúrgica de la pulga en condiciones de asepsia es el tratamiento de elección. Se las debe extraer lo más precozmente posible para evitar infecciones y otras complicaciones secundarias. La extracción del parásito debe ser completa, ya que si quedan restos en el huésped, se puede generar una respuesta inflamatoria local muy importante. Además, se debe indicar vacuna antitetánica en caso de ser necesario ${ }^{5,6}$ y realizar curas locales con antibióticos y antisépticos de las lesiones. Ante múltiples lesiones, se recomien- 
da utilizar ivermectina por vía oral $(200 \mu \mathrm{g} / \mathrm{kg})$. También puede utilizarse niridazole $(30 \mathrm{mg} / \mathrm{kg} /$ día) o tiabendazol ( $25-50 \mathrm{mg} / \mathrm{kg} /$ día). ${ }^{2,7,8}$

La prevención consiste principalmente en la utilización de calzado cerrado y autoexamen diario de los pies para detectar las lesiones incipientes. ${ }^{1}$

\section{CONCLUSIONES}

Debe sospecharse la presencia de tungiasis ante la aparición de lesiones cutáneas negruzcas e hiperqueratósicas, principalmente en zonas periungueales, así como también en plantas, tobillos o talones de personas con antecedentes de haber permanecido descalzas en zonas endémicas de esta parasitosis. Es importante instruir a quienes viajan a dichas zonas, sobre la importancia de usar calzado cerrado para evitar la infestación con este parásito.

\section{BIBLIOGRAFÍA}

1. Menghi CI, Comunale E, Gatta CL. Tungiasis adquirida en la provincia del Chaco, Argentina. Rev Argent Microbiol 2009;41(3):148-50.

2. Escamilla Martínez E, Gómez Martín B, Sánchez Rodríguez R, Martínez Nova A, et al. Tungiasis. Traveler's ectoparasitosis of the foot: a case report. Foot Ankle Int 2008;29(3):354-7.

3. Verdi M, Benavente D, Gentile J, Omaña S, Zusaeta M. Tungiasis. Rev Argent Dermatol 2008;89(4):226-33.

4. Gibbs SS. The diagnosis and treatment of tungiasis. $\mathrm{Br} J$ Dermatol 2008; 159(4):981.

5. Miller H, Rodríguez G. Tungiasis en población indígena del departamento de Vaupés: epidemiología, clínica, tratamiento y prevención. Biomédica 2010;30(2):215-37.

6. Veraldi S, Valsecchi M. Imported tungiasis: a report of 19 cases and review of the literature. Int J Dermatol 2007;46(10):1061-6.

7. Gatti FR, de Olivera CM, Servilha TR, Sánchez APG. Tungíase disseminada tratada com ivermectina. An Bras Dermatol 2008;83(4):339-42.

8. Rosmaninho A, Vilaça S, Costa V, Sarmento A, et al. Tunga penetrans: painful lesions on the feet-the first imported case from Guinea-Bissau. Case Report Med 2010;2010:681302. 